\title{
Pancreatic Stellate Cells: The Conductor of Dissonance in Pancreatic Cancer
}

\author{
Aggelos Margetis*, Dimitris Drekolias, Andreas C. Lazaris \\ Department of Pathology, Medical Faculty, The National and Kapodistrian University of Athens, 11527 Athens, Greece
}

Received: August 16, 2015; Accepted: October 10, 2015; Published: October 29, 2015

*Corresponding author: Aggelos Margetis, Department of Pathology, Medical Faculty, The National and Kapodistrian University of Athens, 75 M. Asias Str. 11527 Athens, Greece, Tel: +30- 210- 7462229, 2267; Fax: +30 210 6644123; E-mail addresses: agelosmrg@yahoo.com

\section{Abstract}

Pancreatic ductal adenocarcinoma (PDAC) has justifiably gained its notoriety. It is the fourth cause of cancer-related death in U.S.A, its incidence is constantly increasing whereas the prognosis is dismal with five year survival rates lower than $5 \%$. These rates are interpreted by the rapid spreading of PDAC and absence of symptoms early in disease. Although surgery can be the only hope for cure, actually, only $10-15 \%$ of patients are candidates for potentially curative surgical resection at presentation. Gemcitabine monotherapy has been the gold standard for locally invasive or metastatic disease while albuminbound paclitaxel (nab paclitaxel) in combination with gemcitabine and the multi-agent FOLFIRINOX (5-fluorouracil, oxaliplatin, irinotecan, leucovorin) are promising novel treatment options. However, the limited clinical response of patients in the above regimens necessitates the discovery of new therapeutic perspectives. Recent studies emphasize the importance of tumor stroma in pancreatic adenocarcinoma.Pancreatic stellate cells (PSCs) are starring in this stroma and, through poorly defined mechanisms, orchestrate a fibroinflammatory reaction which contributes significantly to tumor formation, progression and chemoresistance.This review summarizes current knowledge about the complex interactions between PSCs and other components of tumor and how these affect the clinical course of disease. We also focus on novel therapeutic approaches which result from the increasing understanding of PSCs biology.
\end{abstract}

Keywords: pancreatic adenocarcinoma, pancreatic stellate cells, stroma, desmoplasia

\section{Introduction}

Despite unceasing research therapeutic efforts, mortality rates of pancreatic cancer remain unchanged over 30 years. Difficulty in early imaging detection, lack of specific biomarkers and prominent chemoresistance, all contribute to the devastative nature of PDAC [1]. Failure of existing treatments has shifted research on PDAC pathobiology. Interactions between stromal elements and cancer cells have been proven to be an essential part of cancer inception, growth, progression and immune surveillance.In pancreatic adenocarcinoma, the characteristic histological finding is the dense desmoplastic/fibro-inflammatory reaction surrounding the cancer cells. Pancreatic stellate cells are the prominent cell type in tumor microenvironment and seem to play a crucial role in the formation of this fibrotic stroma [2, 3].

\section{Pancreatic stellate cells: the silent force}

In healthy pancreatic tissue, PSCs are quiescent and are found in minimal numbers, mainly in interlobular and in interacinar regions. Their shape is usually triangular, they have cytoplasmic lipid droplets containing auto-fluorescent vitamin A stored as retinyl palmitate and long cytoplasmic extensions. Their differentiation from fibroblasts results from the expression of vimentin, nestin, desmin, glial fibrillary acid protein (GFAP) as well as neural cell adhesion molecule (NCAM) and nerve growth factor (NGF) [3, 4]. Normally, they are characterized by a low mitotic index and low synthesis capacity of extracellular matrix. However, in the setting of tissue injury, PSCs are activated, lose their vitamin A droplets and adopt a myofibroblast-like phenotype. Growth factors, cytokines and oxidative stress are known to activate PSCs. Among these stimuli, PDGF, ethanol, TGF-a, TGF-b1, hypoxia, sonic hedgehog signaling pathway, activin A, b-FGF, TNF-a, IL-1, IL-6, IL-10, fibrinogen, EMMPRIN, endothelin-1 and angiotensin II are of utmost importance [4, $5,6]$.The major stimuli which activate PSCs are summarized in Figure 1.The activated PSCs, which are indentified by the expression of $\alpha$-smooth muscle actin ( $\alpha$-SMA), demonstrate a high mitotic index, increased migratory properties and an ability to produce large amounts of extracellular matrix proteins. Once activated, they express PDGF receptors, TGF-b receptors and ICAM-1 [4]. The synthesis of collagen types I, III, IV(angiogenesis, invasion, metastasis, chemoresistance), FAP-a(ECM remodelling), TIMP-1/2(matrix degradation, cell survival), fibronectin(cell survival, chemoresistance), decorin (antiproliferative effect toward pancreatic cancer cells), MMP2 \& MMP-9 (ECM degradation, invasion), periostin (cancer cell survival, invasion, chemoresistance), SPARC (invasion, metastasis), tenascin C(invasion, metastasis), thrombospondin-2 (invasion) is increased[7].Growth factors (PDGF, FGF, CTGF, TGF-b1), cytokines (TNF-a, IL-1b, IL-6, IL-8, RANTES, MCP-1) and endothelin-1 are also secreted [4,7].

Interactions with pancreatic cancer cells: an insidious alliance

The interaction between PSCs and neoplastic cells is undoubtedly a complex one involving many different 


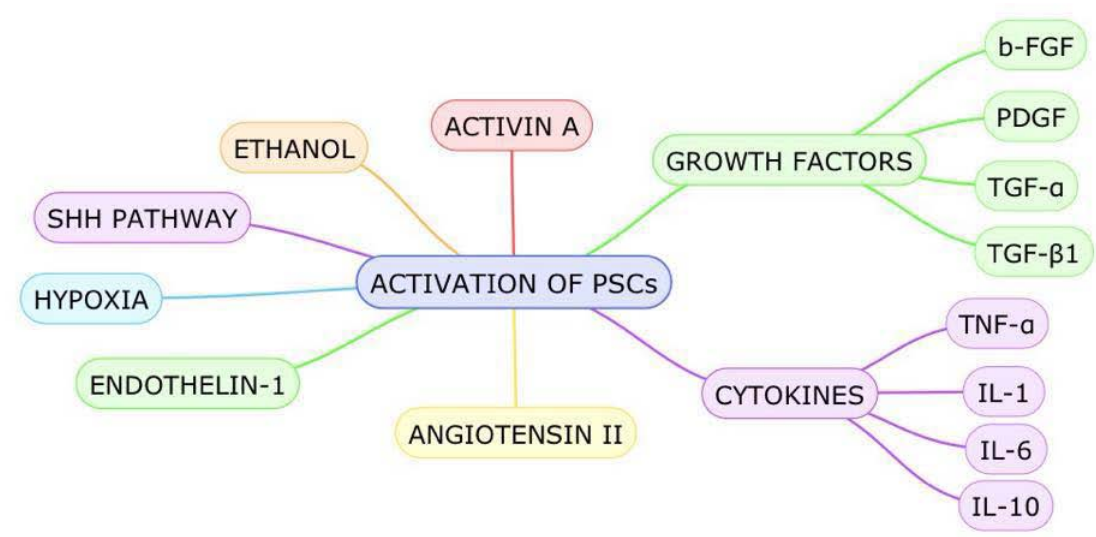

Figure 1: Major stimuli that activate pancreatic stellate cells. Based on the information provided by references.

interconnecting pathways, while the degree of clinical importance of each pathway is yet to be fully revealed. In vivo and in vitro studies have demonstrated that when pancreatic cancer cells are combined with PSCs, the neoplasia evolves more aggressively concerning the size of the tumor, the degree of stromal reaction, the invasive and metastatic potential [6].The TGF- $\beta$ family, strongly related to the PSCs, has been found to be consistently mutated in at least one locus in PDAC patients. PDAC cells stimulate PSCs via secretion of various growth factors, including TGF- $\beta$, HGF, FGF, and EGF, with TGF- $\beta$ being predominant. Another recent study showed that upregulation of COX-2, HAS2, and MMP-1 was found to be associated with increased PDAC invasiveness in a cancer cell-fibroblast co-culture. Additionally, Sonic Hedgehog (SHH) also found to be linked to the desmoplastic reaction around PDAC, stimulating PSCs via a paracrine mechanism, which promotes epithelial-to-mesenchymal transition (EMT), proliferation, and invasive potential. PSCs are known to play a key role in induction of epithelial to mesenchymal transition (EMT). EMT results in cancer cell dissociation from their origin, increased migration and a more malignant phenotype. The expression of membrane-associated $\beta$-catenin, cytokeratin-19 and E-cadherin is shown to be decreased whereas Snail-1 and vimentin expression is increased [8].0ther studies revealed that PDGF secretion from the PDAC cells yield a chemotactic effect on PSCs and that PSCs also inhibit hydrogen peroxide-induced apoptosis, prolonging the PDAC cells survival [9]. Very recently, the relationship of Wnt2 protein that is secreted by the activated PSCs has come to light, showing that increased levels of Wnt2 correlate with enhanced migration, invasion, and metastasis of PDAC cells via activation of the classic Wnt/ $\beta$-catenin pathway [10]. PSCs also promote neoplastic cell invasion via the complex role of FGF family and its receptors. Overexpression of FGF/ FGFR in neoplastic cells has been associated with poor prognosis in PDAC, but the relationship of PSCs and FGF/FGFR has not been thoroughly investigated, although one recent study has enlightened their interplay, suggesting that prevention of FGF/ FGFR mediated proliferation and invasion in PSCs can lead to disruption of the PDAC microenvironment, thus preventing to some degree the invasion of neoplastic cells [11]. Furthermore, the role of micro-RNAs regarding PDAC microenvironment and desmoplasia has also been studied. Upregulation of miR21/ miR221 was found in PSCs of patients with PDAC, compared to PSCs derived from normal pancreas. Moreover, in vitro therapeutic targeting of these miRNAs seemed to be of clinical importance, reducing the likelihood of PDAC progression [12]. The relationship of fibroblast activating protein (FAP) with PDAC stroma and especially PSCs has recently been found to be of considerable prognostic significance, with higher expression of FAP in stage IIB than in stage IIA patients [13]. Additionally, the role of periostin, also known as osteoblast-specific factor 2 , a protein expressed exclusively by the activated PSCs, has been recently suggested to correlate with PDAC increased proliferation and enhanced resistance to starvation and hypoxia, therefore contributing to the PDAC further development [14].Recently, in vivo and in vitro studies tried to evaluate the role of sphingosine1-phosphate (S1P) which is often implicated in tumor growth via yet incompletely understood mechanisms. The studies showed that the enzyme that produces S1P, namely sphingosine kinase 1 , was overexpressed in PDAC cells, leading subsequently to activation of PSCs. The activated PSCs released paracrine factors, including matrix-metalloproteinase-9, which further promoted PDAC cell migration and invasion [15].

\section{Interactions with other stromal components}

Besides the prominent role of PSCs-PDAC interaction in PDAC progression, the complicated cross-talk between PSCs and the other stromal components seems to crucially affect the course of PDAC. The physiologic antitumorigenic immunological response is hindered by the immunosuppressive nature of PSCs. A recent study investigated this immunosuppressive activity, finding increased expression of IP-10/CXCL10 chemokine, induced by the PDAC cells in the PSCs. This overexpression subsequently led to enhanced intratumoral activity of T regulatory cells, with their known immunosuppressive role [16]. In addition, galectin-1 derived from PSCs, was found to enhance apoptosis and anergy of T cells in PDAC patients, further contributing to the escape 
of immune surveillance [17].The association of extracellular matrix (ECM) with the actions of PSCs has also been investigated. Transgelin expression was significantly increased in activated PSCs vs. quiescent PSCs, and silencing of transgelin was linked to decreased PSCs proliferation and migration [18]. Furthermore, the characteristic perineural invasion in PDAC patients, seems to be strongly influenced by the PSCs. Overexpression of Sonic Hedgehog (SHH) in neoplastic cells is an important marker of PDAC biological activity, including perineural invasion. This overexpression stimulates via a paracrine route the hedgehog pathway in PSCs, promoting the migration of the neoplastic cells along nerve axons, therefore contributing to their perineural invasion [19].

\section{Role of PSCs in early carcinogenesis}

Tumor progression into malignant state is characterized by the ability of tumor to invade the surrounding tissue and disseminate via lymphatic and blood vessels. At the earliest stages of carcinogenesis, a distinct stromal reaction around PanIN (pancreatic intraepithelial neoplasia) lesions has been described. This reaction, which comprises extensive collagen deposition and abundant a-SMA positive activated PSCs, led finally to pancreatic cancer development in a mouse model overexpressing Kras [3].Further data, supporting the idea that PSCs are activated early in carcinogenesis process, emanate from the observation of periostin (solely expressed by PSCs) in intraductal papillary mucinous neoplasms of pancreas [3].As mentioned above, PSCs are the main source of MMPs, TIMPs and other proteins that promote metastasis. In particular, expression of the stromal marker SPARC and surface marker CD10 by pancreatic stellate cells stimulates tumor progression and migration [7]. Moreover, there is evidence proposing that PSCs are capable of migrating to the metastasis site early and carrying out an important action, which is to facilitate implantation, survival and proliferation of the metastatic cancer cells [20].In Figure 2, the associations between PSCs, cancer cells and stromal components, all of which contribute to cancer progression, are illustrated.

\section{Chemoresistance and radioresistance in PDAC is mediated by PSCs}

One of the well-documented features of pancreatic cancer is its resistance to chemotherapeutic regimens and to radiotherapy. The dense stromal environment around cancer cells is strongly involved in this resistance. The desmoplasia provides a mechanical protective barrier to the cancer cells as high expression of collagen types I, III, IV, fibronectin, laminin and hyaluronic acid in PDAC stroma correlates with decreased cytotoxicity of anticancer drugs. Activated stellate cells can also express ligands for $\beta 1$-integrins which have been shown to mediate radioresistance in tumor models. Lunardi et al. suggest that inhibition of the $\beta 1$-integrins/FAK signaling pathway is a potential therapeutic strategy to ameliorate the radiosensitivity of PDAC [7]. In addition to this, PSCs appear to contribute to the hypovascular and hypoxic microenvironment found characteristically in pancreatic cancer. The fibrotic reaction facilitates the so-called growth-induced solid stress (GISS)

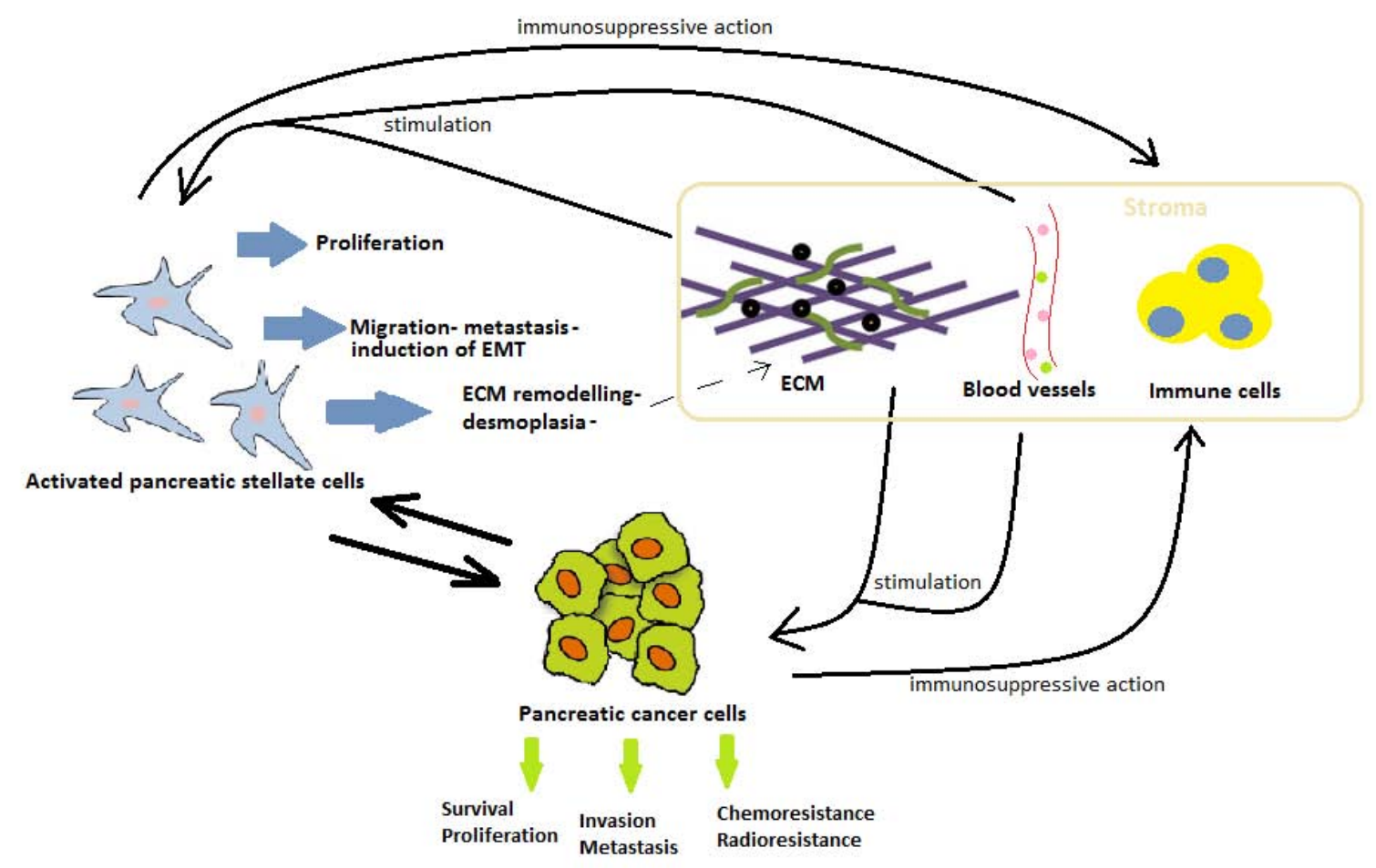

Figure 1: Major stimuli that activate pancreatic stellate cells. Based on the information provided by references. 
resulting in distortion of intratumoral vasculature, which in turn causes hypoxia and increased interstitial pressure; both intensify chemoresistance. Fibrosis is in turn stimulated by hypoxia establishing a self-perpetuating hypoxia-fibrosis cycle. This promotes EMT and genetic instability of cancer cells while at the same time drug delivery is impaired [5]. Importantly, recent publications have demonstrated that PSCs may enhance the stem-like phenotype of cancer stem cells and, through this mechanism, can drive self-renewal and chemoresistance of cancer cell population [21].Finally, Cabrera et al.showed that PSCs survived in vivo in patients treated with full-dose gemcitabine plus concurrent hypo-fractionated stereo-tactic radiosurgery [22]. Indeed, they displayed a more activated phenotype posttherapy. All the above data underlie the significant role of PSCs in rendering the pancreatic cancer refractory in standard nonsurgical therapies.

\section{Targeting PSCs- current and future treatment strategies}

The recently emerging information about the crucial role of PSCs in PDAC pathobiology has offered new potential therapeutic targets. The role of vitamin D receptor ligand calcipotriol has been investigated, through its action as a transcriptional modulator in activated PSCs, reprising their quiescent state, and thus leading to stromal remodelling, more effective gemcitabine chemotherapy, and a $57 \%$ survival prolongation compared to chemotherapy alone [23]. Besides that, retinoic acid has also been utilized, targeting cancer-associated fibroblasts (CAFs), leading to inhibition of PDAC cell migration and EMT via downregulation of IL-6 [24]. Cyclopamide also showed promising results, through its potent inhibitory role in the SHH pathway in PSCs and PDAC cells, resulting in disruption of the stroma and simultaneous enhancement of radiation therapy [25]. Additionally, another publication relying upon the shared characteristics of PSCs with monocyte-macrophage lineage cells, tested nitrogen-containing bisphosphonates as a potential PSCs inhibitory treatment, which were also found to enhance nanoparticle nab-paclitaxel, when combined [26]. Most recently, Kozono et al. showed that administration of pirfenidone, an anti-fibrotic agent, in models of PDAC decreased the growth of tumors through reduction of PSCs activity and through interruption of PCCs and PSCs crosstalk [27]. Angiotensin inhibition has also been proposed as an attractive therapeutic target. Masamune et al. report that olmesartan, an angiotensin IItype 1 receptor blocker, restricted PSCs stimulation and, consequently, tumor progression [28]. Last but not least, nab-paclitaxel or Abraxane has generated great interest. It is postulated that albumin-bound nab-paclitaxel selectively accumulates in pancreatic stroma via its binding to SPARC, acts to deplete PSCs and desmoplastic stroma, enhances intratumoral perfusion and improves drug delivery to tumor tissue [29].

\section{Conclusion}

In conclusion, the complex interactions among PSCs, stromal components, and PDAC cells results in a wide variety of modulatory actions that lead to different pathological outcomes. The prominent role of PSCs in almost all the components of PDAC progression is currently being established by an augmenting number of studies. Further research of the exact interconnecting pathways that clarify the correlation between PSCs and PDAC, will shed light to the obscure nature of this malignancy, providing simultaneously novel therapeutic targets for evaluation.

\section{References}

1. Rossi ML, Rehman AA, Gondi CS. Therapeutic options for the management of pancreatic cancer. World J Gastroenterol. 2014;20(32): 11142-11159. doi: 10.3748/wjg.v20.i32.11142.

2. Vonlaufen A, Phillips PA, Xu Z,et al. Pancreatic stellate cells and pancreatic cancer cells: an unholy alliance. Cancer Res. 2008; 68(19):7707-7710.doi: 10.1158/0008-5472.CAN-08-1132.

3. Wilson JS, Pirola RC, Apte MV. Stars and stripes in pancreatic cancer: role of stellate cells and stroma in cancer progression. Front Physiol. 2014; 5:52. doi: 10.3389/fphys.2014.00052. eCollection 2014.

4. Bachem MG, Zhou Z, Zhou S, Siech M. Role of stellate cells in pancreatic fibrogenesis associated with acute and chronic pancreatitis. J Gastroenterol Hepatol. 2006;21(3): S92-96.

5. McCarroll JA, Naim S, Sharbeen G, Russia N, Lee J, Kavallaris M, et al. Role of pancreatic stellate cells in chemoresistance in pancreatic cancer. Front Physiol. 2014;5:141. doi: 10.3389/fphys.2014.00141. eCollection 2014.

6. Haqq J, Howells LM, Garcea G, Metcalfe MS, Steward WP, Dennison AR. Pancreatic stellate cells and pancreas cancer: current perspectives and future strategies. Eur J Cancer. 2014;50(15):2570-2582. doi:10.1016/j.ejca.2014.06.021.

7. Lunardi S, Muschel RJ, Brunner TB. The stromal compartments in pancreatic cancer: are there any therapeutic targets? Cancer Lett. 2014;343(2):147-155.doi: 10.1016/j.canlet.2013.09.039.

8. Kikuta K, Masamune A, Watanabe T, Ariga H, Itoh H, Hamada S et al. Pancreatic stellate cells promote epithelial mesenchymal transition in pancreatic cancer cells. Biochem Biophys Res Commun. 2010;403(34):380-384. doi: 10.1016/j.bbrc.2010.11.040.

9. Moir JA, Mann J, White SA. The role of pancreatic stellate cells in pancreatic cancer. Surg Oncol. 2015. doi:10.1016/j. suronc.2015.05.002.

10. Xu Y, Li H, Huang C, Zhao T, Zhang H, Zheng C et al. Wnt2 protein plays a role in the progression of pancreatic cancer promoted by pancreatic stellate cells. Med Oncol. 2015;32(4):97. doi:10.1007/s12032-0150513-2.

11. Coleman SJ, Chioni A-M, Ghallab M, Anderson RK, Lemoine NR, Kocher HM, et al. Nuclear translocation of FGFR1 and FGF2 in pancreatic stellate cells facilitates pancreatic cancer cell invasion. EMBO Mol Med. 2014;6(4):467-481.doi:10.1002/emmm.201302698.

12. Ali S, Suresh R, Banerjee S, Bao B, Xu Z, Wilson J, et al. Contribution of microRNAs in understanding the pancreatic tumor microenvironment involving cancer associated stellate and fibroblast cells. Am J Cancer Res. 2015;5(3):1251-1264.

13. Patsouras D, Papaxoinis K, Kostakis A, Safioleas MC, Lazaris AC, Nicolopoulou-Stamati P.Fibroblast activation protein and its prognostic significance in correlation with vascular endothelial growth factor in pancreatic adenocarcinoma. Mol Med Rep. 2015 
Jun;11(6):4585-90. doi: 10.3892/mmr.2015.3259.

14. Liu Y, Du L. Role of pancreatic stellate cells and periostin in pancreatic cancer progression. Tumour Biol. 2015;36(5):3171-3177. doi:10.1007/s13277-015-3386-2.

15. Bi Y, Li J, Ji B, Kang N, Yang L, Simonetto DA, et al. Sphingosine-1phosphate mediates a reciprocal signaling pathway between stellate cells and cancer cells that promotes pancreatic cancer growth. Am J Pathol. 2014;184(10):2791-2802. doi:10.1016/j.ajpath.2014.06.023.

16. Lunardi S, Jamieson NB, Lim SY, Griffiths KL, Carvalho-Gaspar M, AlAssar 0, et al. IP-10/CXCL10 induction in human pancreatic cancer stroma influences lymphocytes recruitment and correlates with poor survival. Oncotarget. 2014;5(22):11064-11080. Available at: http:// www.ncbi.nlm.nih.gov/pubmed/25415223.

17. Tang D, Gao J, Wang S, Yuan Z, Ye N, Chong Y, et al. Apoptosis and anergy of $\mathrm{T}$ cell induced by pancreatic stellate cells-derived galectin-1 in pancreatic cancer. Tumour Biol. 2015;36(7):5617-5626. doi:10.1007/s13277-015-3233-5.

18. Apte M V, Yang L, Phillips PA, Xu Z, Kaplan W, Cowley M, et al Extracellular matrix composition significantly influences pancreatic stellate cell gene expression pattern: role of transgelin in PSC function. Am J Physiol Gastrointest Liver Physiol. 2013;305(6):G408-417. doi:10.1152/ajpgi.00016.2013.

19. Li X, Wang Z, Ma Q, Xu Q, Liu H, Duan W, et al. Sonic hedgehog paracrine signaling activates stromal cells to promote perineural invasion in pancreatic cancer. Clin Cancer Res. 2014;20(16):4326-38. doi:10.1158/1078-0432.CCR-13-3426.

20.Xu Z, Pothula SP, Wilson JS, Apte MV. Pancreatic cancer and its stroma: a conspiracy theory. World J Gastroenterol. 2014;20(32): 1121611229. doi: 10.3748/wjg.v20.i32.11216.

21. Lonardo E, Frias-Aldeguer J, Hermann PC, Heeschen C. Pancreatic stellate cells form a niche for cancer stem cells and promote their selfrenewal and invasiveness. Cell Cycle. 2012;11(7): 1282-1290. doi: 10.4161/cc.19679.

22. Cabrera MC, Tilahun E, Nakles R, Diaz-Cruz ES, Charabaty A, Suy
S, et al. Human Pancreatic Cancer-Associated Stellate Cells Remain Activated after in vivo Chemoradiation. Front Oncol. 2014; 4: 102. doi: 10.3389/fonc.2014.00102. eCollection 2014

23. Sherman MH, Yu RT, Engle DD, Ding N, Atkins AR, Tiriac H, et al. Vitamin D Receptor-Mediated Stromal Reprogramming Suppresses Pancreatitis and Enhances Pancreatic Cancer Therapy. Cell. 2014;159(1):80-93. doi:10.1016/j.cell.2014.08.007.

24. Guan J, Zhang H, Wen Z, Gu Y, Cheng Y, Sun Y, et al. Retinoic acid inhibits pancreatic cancer cell migration and EMT through the downregulation of IL-6 in cancer associated fibroblast cells. Cancer Lett. 2014;345(1):132-139. doi:10.1016/j.canlet.2013.12.006.

25.Zhao J, Wu C, Abbruzzese J, Hwang RF, Li C. Cyclopamine-loaded core-cross-linked polymeric micelles enhance radiation response in pancreatic cancer and pancreatic stellate cells. Mol Pharm. 2015;12(6):2093-2100. doi:10.1021/mp500875f.

26. Gonzalez-Villasana V, Rodriguez-Aguayo C, Arumugam T, CruzMonserrate Z, Fuentes-Mattei E, Deng D, et al. Bisphosphonates inhibit stellate cell activity and enhance antitumor effects of nanoparticle albumin-bound paclitaxel in pancreatic ductal adenocarcinoma. Mol Cancer Ther. 2014;13(11):2583-2594. doi:10.1158/1535-7163.MCT14-0028.

27. Kozono S, Ohuchida K ,Eguchi D ,Ikenaga N ,Fujiwara K, Cui L, et al. Pirfenidone inhibitspancreatic cancer desmoplasia by regulating stellate cells. Cancer Res . 2013;73(7):2345-2356. doi: 10.1158/00085472.CAN-12-3180.

28. Masamune A, Hamada S, Kikuta K, Takikawa T, Miura S, Nakano $\mathrm{E}$, et al. The angiotensin II type I receptorblocker olmesartan inhibits the growth of pancreaticcancer by targeting stellate cell activities in mice. Scand JGastroenterol 2013;48(5):602-609. doi: 10.3109/00365521.2013.777776.

29. Von Hoff DD, Ramanathan RK, Borad MJ, Laheru DA, Smith LS, Wood $\mathrm{TE}$, et al. Gemcitabine plus nab-paclitaxel is an active regimen in patients with advanced pancreatic cancer: a phase I/II trial. J Clin Oncol. 2011;29(34): 4548-54. doi: 10.1200/JC0.2011.36.5742. 